International Journal of Engineering \& Technology, $7(2.19)(2018)$ 104-105
International Journal of Engineering \& Technology
WPC
Website: www.sciencepubco.com/index.php/IJET
Research paper

\title{
Descry and Divulge Hitch by Using Hitch Entrapment-Bug Catcher
}

\author{
J.Vivek $^{1^{*}}$, B.Hema ${ }^{2}$, J.Apoorva ${ }^{3}$ \\ ${ }^{1,}$ Assistant Professor \\ Department Of Computer Science And Engineering, School Of Computing, \\ Vel Tech Rangarajan Dr. Sagunthala R\&D Institute Of Science And Technology, \\ Avadi, Chennai-600 062, Tamil Nadu, India. \\ ${ }^{, 2,3}$ ug Scholar \\ Department Of Computer Science And Engineering, School Of Computing, \\ Vel Tech Rangarajan Dr. Sagunthala R\&D Institute Of Science And Technology, \\ Avadi, Chennai-600 062, Tamil Nadu, India. \\ *Vivekjustus@Gmail.Com
}

\begin{abstract}
Based on the recent survey they used to track the bugs which are stored for further uses. The system is updated with new data by the technician. To overcome this situation hitch entrapment is proposed to make testing session productive and record the details to aid the development team for quick Resolution. This work is associated with developer, tester and admin. This tool allows the developer and tester to post the queries and screenshots of their program and Admin will monitor the whole process..
\end{abstract}

Keywords: Bug, Testing

\section{Introductionl}

It is clutter free user interface. Quick and easy to make bug reporting directly into the system and remove dependency on spreadsheet this tool provide simple status update for smooth communication between testing and developing team. This tool is a primary input to the development team to architect a solution for the project. This is very simple and easy to understand and user can directly interact with the devices. It will provide a simple update between the tester and developing team for the better communication. The displayed list is valid only for the logged in user. Thus the user has rights which are displayed along with the bugs reported by the user. The row expands to display the list of bugs . The bug titles are displayed with

status of closed or work in progress .User may choose this requirement to test again and post the verified status on the bug status. The system will mark "closed", if once all the bugs have been fixed and verified for a test case. The test cases for which no bugs were reported, will require the user to manually set the status as closed.

\section{Literature Survey}

[1]It is the process of executing a program with the intent of finding errors. It concentrates on the testing and assessing the smallest piece of the underlying software.

[2] Bug tracking system provides an "automated questionnaire system" which collects all the information on behalf of the tester.
[3] With the goal of discovering software bugs, The test methods incorporate the way towards executing a program or application and confirming that the software item is ready for utilizing. To assess at least one property of intrigue the software testing includes the execution of framework component.

[4] It presents the pros and cons of Automation and also manual testing helps us to understand the overall testing behavior for project scalability and reliability.

\section{Existingsystem}

\subsection{Outlook:}

In present the existing system user does not specify all the information needed for tester .It is used to generate report on the productivity of programmers by fixing bugs. This may sometime yield to inaccurate result because different bugs may have different severity and complexity.

\subsection{SEQUENCE OF PROCEDURE}

${ }^{[2]}$ Initially, we need the exact location of the bug in the program. Location gives you the information like line number of the bug in the program, its class and so on. This helps the developer to locate the code. Bug tracking system database records the facts of the known bug. Then it identifies the person who reported it or any programmers who may be working on it. 


\section{Proposed System}

\subsection{Introduction}

BUG catcher tool for a leading software company. The user will access bug catcher application already bookmarked in the browser and capture the following details for a project.

- URL of the page where the bug was found

- Attach a snapshot of the error page.

- $\quad$ Select the test case, enter the brief title, select severity (shows stopper, high, and medium, low) and post the bugs.

\subsection{Outlook}

Hitch entrapper integrated with the software companies intranet. It uses only the login and authentication mechanism of intranet. This tool allows the testing team to post the bugs and development team to access the information.

\subsection{Sequence of Procedure}

1. Landing Page:-

The landing page of the project the project displays and count the bugs reported and resolved .List displayed is valid only for the logged in user choose the query and resolve it and post it the verified bugs more out of the list.

2. Access Bug Report:-

The development team can be able to access the bug record from the landing page. If they view the bug the user can click and open the bug record and fix the issue offline in the development environment .The development users post the status of the bug as "Resolved".

\subsection{Flowchart}

- At, first the admin should register the developer.

- Then the admin will post the screenshots of the program.

- The queries will be stored in view query.

- Then it is viewed by the tester and starts to test the program.

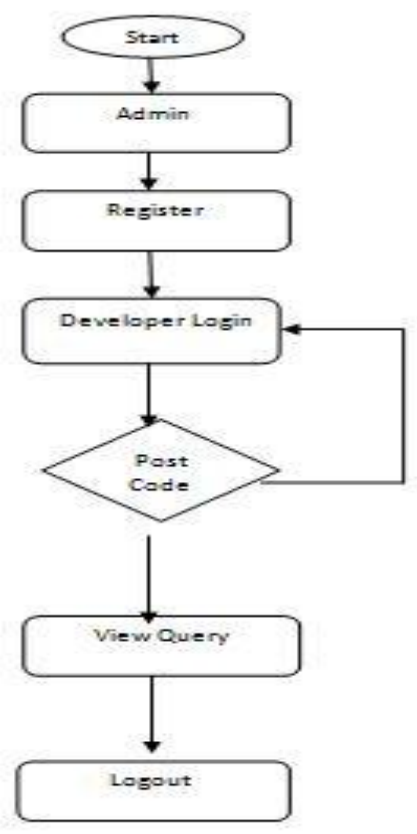

Fig 1. Flowchart
Tester:

- At first, the admin should register the Tester.

- Tester will view the query from view query and post it response query.

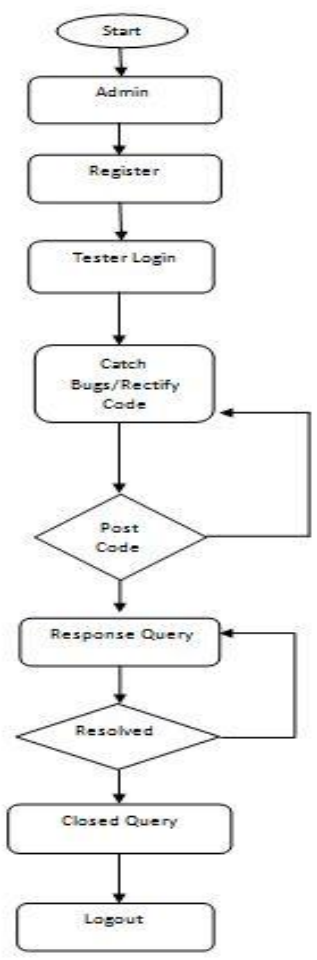

Fig 2 Tester Process

\section{CONCLUSION}

The suggested tool is used to track the bugs and the tester have to test .The tester has to be physically present .The proposed system will be a compact tool which can be used by the developers and testers to reduce the time, where persons are not required physically. In future the bugs will be automatically solved.

\section{Acknowledgement}

The work is carried out under the supervision of J.VIVEK (Assistant Professor), School of Computing, Veltech Rangarajan Dr.Sagunthala R \& D Institute Science and Technology. We express our heart full thanks.

\section{References}

[1]. Umesh Kumar Tiwari, Santosh Kumar "Component Level Testing in Component-Based Software Development" International Journal of Innovations \& Advancement in Computer Science IJIACS ISSN 2347 - 8616 Volume 6, Issue 1 January 2017.

[2]. Syed Parvez1, Syed Yousef Danish Mehdi2, Md Sufiyan Ali3, Prof Shaik Maheboob4, Defect Tracking System, International Journal for Research in Applied Science \& Engineering Technology (IJRASET) Volume 5 Issue III, March 2017 IC Value: 45.98 ISSN: 2321-9653

[3]. Monika Thakur, Sanjay, "Review on Structural Software Testing Coverage Approaches", International Journal for Advance Research ideas and innovations in Technology, ISSN: 2454-132X Impact factor: 4.295 (Volume3, Issue3), @2017.

[4]. D. Bhatt1 ," A Survey of Effective and Efficient Software Testing Technique and Analysis", L.J.Institute of Engineering and Technology,@ July 2017 | IREJOURNALS | Volume 1 Issue 1 | ISSN: Applied. 